Erfahrungsbericht:

\title{
Praktische Umsetzung des Informationsmanagements
}

\section{Vier Jahre Abteilung Informations- management an der Gesamthoch- schulbibliothek Kassel}

Dr. Helge Steenweg

\section{Informationsmanagement}

Im Bibliothekswesen ist ein allgemeiner Umbruch zu verzeichnen. Sehr deutlich zeichnet sich infolge des steigenden Informationsbedarfes im akademischen wie auch im kommerziellen Bereich eine Umorientierung der zentralen universitären Einheiten, wie Bibliotheken und Rechenzentren, ab. Immer stärker muß daher der Dienstleistungsfaktor in die Entwicklung dieser Betriebseinheiten einbezogen werden. Dies bedeutet selbst in Zeiten knapper Personalressourcen für Bibliotheken und Rechenzentren eine Ausweitung der Dienstleistungen. Für die Bibliotheken folgt weiter, daß die bisherige Aufgabe, Schrifttum im weiteren Sinne zu sammeln, zu archivieren und der Leserschaft zur Verfügung zu stellen, nicht mehr genügt, um das steigende Informationsbedürfnis der Benutzer zu befriedigen und sich den in rascher Folge erneuernden "Neuen Medien" zu stellen. Bibliotheken werden daher in mittelfristiger Zeit zu Informationsvermittlungsstellen werden, die den Benutzern im großen Maßstab die gewünschten und benötigten Informationen rasch zur Verfügung stellen. Sie werden sich dabei immer mehr neuester Technologien bedienen müssen. Diese Entwicklungen wurden politisch bereits frühzeitig erkannt und in verschiedenen wegweisenden Empfehlungen schriftlich fixiert. Insbesondere die mit DV-Technik befaßten Gremien der Deutschen Forschungsgemeinschaft (DFG) haben sich mit dieser Thematik in verschiedenen Ausschüssen befaßt[1].

\section{Entwicklung Kassel}

Das Bibliothekssystem in Kassel hat als einschichtige Universitätsbibliothek der Gesamthochschule Kassel (GHK), als Hessische Landesbibliothek und als wissenschaftliche Stadtbibliothek (Murhard) die Literaturversorgung wie auch Informationsvermittlung für Bürger, Studenten und Wissenschaftler in Nordhessen, insbesondere in der Region Kassel, aufzubauen. An Eckwerten ist zu nennen (1997): 1,5 Mio. Buchbestand, 3,025 Mio. DM Normaletat (davon 2,64 Mio DM Bucherwerbungsgelder), 114 Personalstellen, 0,8 Mio jährliche Entleihungen (incl. Verlängerungen)[2].

Seit 1993/94 wurde seitens der Hochschule verstärkt eine Modernisierung der Gesamthochschulbibliothek (GHB) gefordert und betrieben. Dies führte zur Einrichtung von Bereichsbibliotheken und zu einer Reorganisation des Bibliotheksbetriebes. Im Rahmen dieser Bibliotheksreorganisation wurde am 1.3.1995 aus der bisherigen Abteilung I (Bibliotheks-DV) durch Zuweisung von drei weiteren Stellen des höheren Dienstes die Abteilung Informationsmanagement (I'M) begründet.

\section{Personalentwicklung}

Der Personalstand der Abt. I'M war einigen Veränderungen ausgesetzt. Tabellarisch sieht die Entwicklung der Abteilung wie folgt aus:

$\begin{array}{lrrrr} & \mathbf{1 9 9 4} & \mathbf{1 9 9 5} & \mathbf{1 9 9 7} & \mathbf{1 9 9 8} \\ \text { Bibl. Höherer Dienst } & 1,0 & 1,0 & 1,5 & 1,0 \\ \text { Bibl. Gehobener Dienst } & 1,5 & 1,5 & 2,5 & 2,5 \\ \text { Bibl. Mittlerer Dienst } & 1,0 & 1,0 & 1,0 & 1,0 \\ \text { Zw.-Summe Bibl. } & 3,5 & 3,5 & 5,0 & 4,5 \\ & & & & \\ \text { DV Höherer Dienst } & & 3,0 & 2,5 & 1,5 \\ \text { DV Gehobener Dienst } & & & & \\ \text { DV Mittlerer Dienst } & 1,0 & 1,0 & 1,0 & 1,0 \\ \text { Zw.-Summe DV } & 1,0 & 4,0 & 3,5 & 2,5 \\ & & & & \\ \text { Zeit. Höherer Dienst } & & & 1,0 & 1,0 \\ \text { Zeit. Gehobener Dienst } & & & & 0,5 \\ \text { Zeit. Mittlerer Dienst } & & & 1,0 & 1,5 \\ \text { Zw.-Summe Zeitstellen } & & & & \\ & 4,5 & 7,5 & 9,5 & 8,5 \\ \text { Summe } & & & & \\ & & & \end{array}$

Deutlich treten die Verschiebungen im Höheren und Gehobenen Dienst hervor:

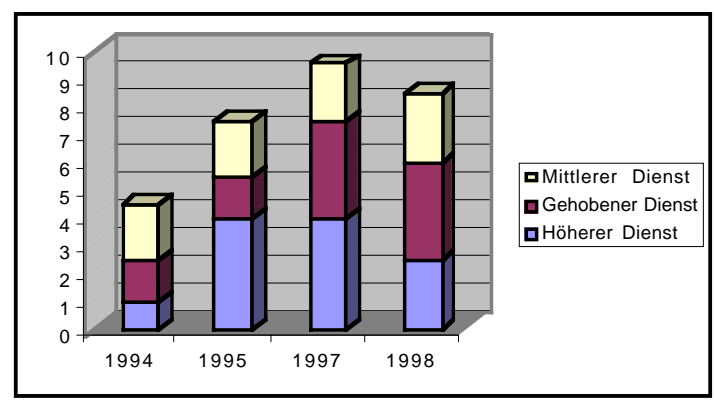

Abb. 1: Stellenentwicklung der Abt. I'M 1994-98

Schwerwiegend ist die Entwicklung, daß trotz eines starken Anwachsens an Aufgaben die Personalentwicklung nicht im gleichen Maße fortgeschrieben werden konnte. Es wurde versucht, dies über Zeitstellen auszugleichen

$\begin{array}{lrrrr} & \mathbf{1 9 9 4} & \mathbf{1 9 9 5} & \mathbf{1 9 9 7} & \mathbf{1 9 9 8} \\ \text { Höherer Dienst } & 1,0 & 4,0 & 4,0 & 2,5 \\ \text { Gehobener Dienst } & 1,5 & 1,5 & 3,5 & 3,5 \\ \text { Mittlerer Dienst } & 2,0 & 2,0 & 2,0 & 2,5 \\ & & & & \\ \text { Summe } & 4,5 & 7,5 & 9,5 & 8,5\end{array}$

Grafisch dargestellt wird der hohe Anteil von Zeitstellen noch deutlicher. Diese Zeitstellen wurden nicht über hochschuleigene Mittel finanziert, sondern über eingeworbene Projektgelder bezahlt. Voraussichtlich wird sich diese Entwicklung in 1999 nicht fortsetzen. Bei gewachsenen Aufgaben hat es zwischen 1995 und 1998 eine deutliche Reduzierung und Verlagerung der Stellendefinitionen (Anteil von Zeitstellen, Verringerung der festen DV-Stellen) gegeben.

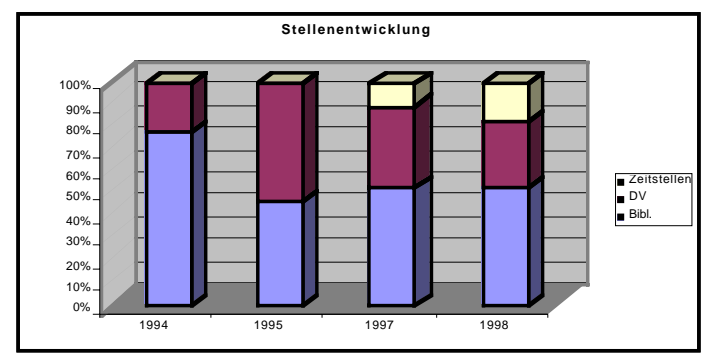

Abb. 2: Stellenentwicklung der Abt. I'M 1994-98 in Prozent 


\section{Etat-Entwicklung}

Die Etat-Entwicklung der Abt. I'M stellt sich demgegenüber zufriedenstellender dar. Es war und ist akzeptiert, daß die Förderung Neuer Medien und deren Umsetzung den Einsatz entsprechender Geldmittel erfordern. Zusätzlich wurde versucht, Drittmittel und Projektgelder einzuwerben. Die Grafik zeigt die leichten Schwankungen im Grundetat ab dem Jahr 1996. In den Jahren 1995/96 kamen etatverstärkend die für Kassel vom Hochschulrechenzentrum (HRZ) und der Bibliothek beantragten HBFG-Gelder für die PICA-Migration in Höhe von ca. 1 Mio. DM hinzu. Der Anstieg im Jahre 1998 ergibt sich durch die von der Abt. I'M verhandelten, organisierten und abgerechneten Landesmaßnahmen und -Projekte.

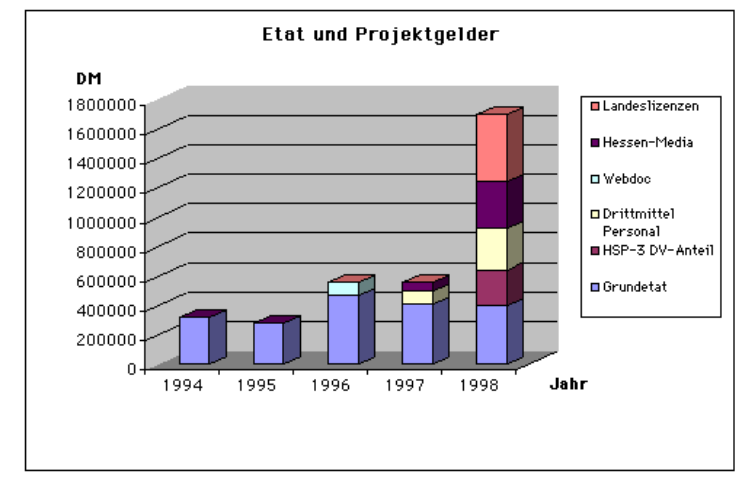

Abb. 3: Etat-Entwicklung

\section{DV-Entwicklung}

\section{Stand 1994}

Die Ausstattung der damaligen Bibliotheksdatenverarbeitung bezüglich Ausleihsystem BIBDIA bestand 1994/95 aus einem Terminalrechner (Norsk Data 5400 Compact) und einem Datenbankrechner (ND TP-Server), auf denen unter dem Betriebssystem SINTRAN III Datenbankanwendungen auf SIBAS-Ebene abliefen. Über Modem- und Standleitungen hingen 26 Tandberg-Terminals an diesem Rechnersystem, jeweils gekoppelt mit Arbeitsplatzdruckern (Nadeldrucker), Magazindruckern und Systemdruckern.

Der CD-Server der GHB bestand aus einem Novell-Server (PC 80486/33), auf dem die CD-Manager-Software der Fa. $\mathrm{H}+\mathrm{H}$ installiert war. Verbunden mit dem Server waren zwei Meridian-Türme mit der Kapazität von 14 bzw. 7 CD‘s. Benutzbar war dieses System von den in der Bibliothek aufgestellten CD-PC's. In der Bibliothek waren insgesamt 28 PC's verschiedener Baureihen (PC 80286, 80386, 80486) vorhanden, die allesamt untereinander unvernetzt aufgestellt waren. Einige PC's verfügten über TCP/IP-Zugriff auf das Campus-Netz bzw. über Modem-Verbindungen zu OnlineAnbietern. Zwei PCs verfügten über einen anonymen $\mathrm{Zu}$ gang ins Internet. Auf dem Gopher-Server des HRZ betrieb die Bibliothek Gopher-Seiten, um interessierten Benutzern über Gopher-Clients Informationen zur Bibliothek zu vermitteln.

\section{Entwicklung ab 1995}

Mit Gründung der Abt. I'M wurde versucht, aus dem bisherigen Bestand an DV-Anlagen eine den gestiegenen Anforderungen der Benutzer gerechte Ausstattung zu schaffen. Als erste Maßnahme dazu wurde ein Konzept entwickelt, das die anzuschaffenden Mitarbeiter- und Benutzer-PC's effizient und arbeitsökonomisch zusammenfaßte. Da allein die zukünttige Anzahl der PC's eine separate Aufstellung jedes $P C$ 's unmöglich machte, war klar, daß nur mit einem Netzbetriebssystem Software- und Hardware-Wartung von dem vor- handenen Personal geleistet werden konnte. Die Entscheidung fiel auf das damals relativ neue Windows NT. Mittlerweile werden ca 300 Bibliotheks-PC's über NT-Server administriert incl. Netzdiensten wie eigenem Mail-Server etc. Auf den jeweiligen Benutzer-PC's wurde Windows NT Workstation installiert, um trotz umfangreicher Arbeitsmöglichkeiten dennoch ein hohes $M a ß$ an Netzwerk-Sicherheit gewährleisten zu können.

Um eine einheitliche Grundlage für die weitere Betreuung von DV-Servern zu schaffen, fiel bei der Auswahl des neuen CD-Servers 1995 die Wahl auf die UltraNet-Software, da die Herstellerfirma damals als einzige eine CD-Server-Software unter Windows NT und ein Caching von CD-Inhalten anbieten konnte. Ein Jahr später erfolgte die Installation eines Silverplatter-Servers auf SUN-Plattform, gefolgt von einem Knight-Ridder-WWW-Server (Dialog) auf NT-Basis.

Mit der Einführung des PICA-Systems in Hessen 1995 waren die Bibliotheken gehalten, ihre zukünftigen Lokalsysteme mit Rechnerkapazität auszustatten. In Rücksprache mit dem Hochschulrechenzentrum, das für die hard- und betriebssystemseitige Betreuung dieser Rechner verantwortlich zeichnet, wurden 1995 zwei Server der Firma DEC angeschafft. Im Jahre 1996 konnte das PICA-Cluster um einen dritten Testserver erweitert werden.

Die softwareseitige Betreuung mit Sybase-DB und LBS-Software erfolgt durch die Abt. I'M der GHB. Hier ist auch die eigentliche Anpassung (Parametrisierung) der lokalen PICAModule angesiedelt. Entscheidend verbessert hat sich für die PICA-Nutzer die Benutzerschnittstelle. Seit 1997 wird neben dem alten Telnet-OPAC im hessischen Bibliotheksverbund der sog. WebOPAC angeboten. Auch die Benutzerdaten können mit der entsprechenden Berechtigung nunmehr im WebOPAC abgefragt werden. Eine Mail-Schnittstelle wurde in den WebOPAC der GHB eingebaut, damit sich Benutzer die Rechercheergebnisse auf die eigene E-Mail-Adresse zuschicken lassen können. Die Fernleihkomponente wurde als letztes PICA-Modul Ende 1998 in Betrieb genommen.

Durch die HBFG-geförderte PICA-Maßnahme und bewilligte Drittmittelanträge für wissenschaftliche Projekte hat sich die DV-Infrastruktur an der GHB gegenüber 1995 grundlegend geändert, was sich auch in neuen oder erweiterten Diensten und Dienstleistungen für Benutzer sichtbar niederschlägt. Die Konfiguration der wesentlichen Hardware-Komponenten der GHB stellt sich wie folgt dar.

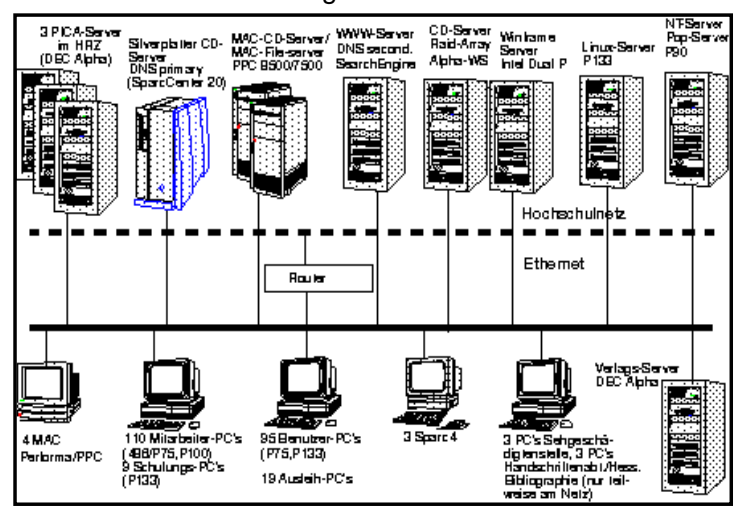

Abb. 4: Hardwarekonfiguration 1998

\section{Aufgaben der Abteilung I'M}

Wie die Namensgebung bereits andeutet, hat diese Abteilung über die DV-Betreuung der Bibliothek hinaus einen deutlich erweiterten Aufgabenzuwachs erhalten (Überblick):

- Bibliotheks-Server (PICA LBS-System). Schon 1994 hatte eine Expertenrunde an der GHK eine Teilung der zukünftigen Bibliotheks-DV-Aufgaben vorgenommen. Im Rahmen der PICA-Migration wurden drei DEC-Worksta- 
tions erworben, die hardware- und betriebssystemseitig vom HRZ, anwendungsseitig (PICA-Software: LBS, Sybase) von der Abteilung l'M betreut werden.

\section{- Bibliotheks-Datenverarbeitung. Im Jahre 1995} benutzten nur die Ausleihe und Katalogisierung übergeordnete DV-Hilfsmittel (BIBDIA, Hebis96). Mit der Einführung von PICA nutzen nunmehr fast alle Bereiche der GHB DV-Kapazitäten.

- Bibliotheksnetz. Von ehemals ca. 25 PC's im Jahre 1995 vergrößerte sich der PC-Bestand um fast den Faktor 12. Über 300 PC's und Workstations werden heute von dieser Abteilung betreut. Dieses unter Windows NT administrierte PC-Netz ist damit zu einem der größten zusammenhängenden PC-Pools auf dem Campus geworden. Die Betreuung schließt die Administration (NT-Server) und Koordination von Netzdiensten (Mailserver, DNS für Verlag) mit ein. Die physikalische Netzplanung in der Bibliothek obliegt wie auch an den anderen Hochschuleinrichtungen und Fachbereichen dem HRZ, die softwaremäßige Planung (TCP/IP, Netzdienste wie Mailserver, DNS, Fileserver, WWW-Server etc.) leistet die Abt. l'M selbst.

- CD-Server. Über einen Windows-NT-Server können sämtliche PC's auf dem Campus mit TCP/IP-Zugriff in den Datenbanken der GHB recherchieren. Dazu ermöglicht ein Winframe-Server auch Nutzern anderer Rechnerplattformen (UNIX-Derivate, MAC) über das Hochschulnetz auf diese Dienste zuzugreifen. Die Abteilung betreut die Rechner hardware- und softwareseitig. Innerhalb der Bibliothek werden die CD-Käufe von der Abteilung koodiniert. Auf dem SUN-Cluster der Bibliothek wurde ein Silverplatter-Server für die Bereitstellung Client-Server-basierter Datenbanken eingerichtet. Dieser Server ist ebenso wie die Knight Ridder-Datenbank über das Internet erreichbar.

- Online-Dienste. Im Rahmen der Informationsvermittlung erruiert die Abteilung Kosten für die Nutzung von Fachinformationsdiensten und koodiniert Verträge für die GHB und landesweite Maßnahmen.

- luK-Initiative. Zur besseren Information und Koordination wurde unter Federführung von Prof. Dr. B. Fricke ein Arbeitskreis "Informationsvermittlung" ins Leben gerufen, den die Abt. I'M betreut. Dieser Arbeitskreis wird regelmäßig über die landesweiten Maßnahmen informiert und trifft eine Vorauswahl über die CD- und Online-Anschaffungen der Hochschule.

- WWW-Server Bibliothek. Auf dem bibliothekseigenen SUN-Cluster ist für die Silverplatter-Installation ein eigener HTTP-Server installiert worden. Der Online-Verlag Kassel-University-Press (KUP) verfügt ebenfalls über einen eigenen WWW-Server auf einer Alpha unter NT sowie über entsprechende DNS-Services auf Intel- und SUN-basierten Rechnern. Der Verlag wurde von der Abt. I'M inhaltlich und hardwaremäßig für die GHK betreut.

Die IntraNet-Seiten der Bibliothek werden in der Abteilung bearbeitet (Umsetzung von Protokollen, Sitzungsniederschriften, Mitteilungen etc.). Es erfolgt ebenso eine Unterstützung der Abteilungen bei der Web-SeitenGestaltung (Grafik, HTML).

- Ausbildung der Mitarbeiter. Durch den massiven DVEinsatz in der Bibliothek fiel ein erheblicher Bedarf an PC-Schulungen an, der größtenteils intern von der Abteilung l'M abgedeckt wurde. Fast alle 150 Mitarbeiter erhielten Schulungen zu Betriebssystemen, Netzanwendungen und Internet. Seit 1996 folgten Spezial-Schulungen zum Einsatz von Windows, Applikaionssoftware und elektronischer Kommunikation.

- Koordinierungsaufgaben im PICA-Projekt, Pilotanwender. Im Rahmen der PICA-Migration beteiligte sich die Abteilung I'M an der hessenweiten Koordinierung der Ausleih-Migration von BIBDIA zu PICA. Für die betreffenden hessischen UB's war die GHB mit der StUB Frankfurt Pilotanwender für die Datenmigration der BIBDIA-Ausleihdaten und ab dem 1.Q. 1997 hessischer Pilotanwender für die PICA-Erwerbung.

- Zeitschriftenerfassung. Eine Mitarbeiterstelle (mittlerer Dienst) ist für die Erfassung der Zeitschriften und Sondermedien in PICA zuständig. Die Herstellung von Buchetiketten für die Erwerbungsabteilung ist hier ebenfalls angesiedelt.

\section{PICA-Lokalsystem}

Im Lande Hessen hat die DV-Entwicklung bei den Bibliotheken durch die Einführung des Bibliothekssystems PICA einen nicht unerheblichen Schub genommen. Dabei kamen auch hier wieder die Empfehlungen der Kommission für Rechenanlagen der DFG "Zur Ausstattung der Hochschulen in der Bundesrepublik Deutschland mit Datenverarbeitungskapazität für die Jahre 1992 bis 1995“ zur Geltung, die bereits 1991 festlegten[1]:

Die Anwendersysteme sollen durch Nutzung von Stan-

dardsoftware oder durch Übernahme marktgängiger

Systeme realisiert werden, wobei im Hinblick auf die Porta-

bilität der UNIX-Kompatibilität der Vorzug zu geben ist.

Wegen der außerordentlichen hohen Entwicklungskosten

sollen höchstens zwei bis drei konkurrierende Standardsoft-

waresysteme im HBFG-Verfahren finanziell gefördert wer-

den.

Alle Bibliothekssysteme sind in das Computernetz der

Hochschule mit dem Ziel zu integrieren, vom Arbeitsplatz-

rechner der Wissenschaftler und der Studenten aus Dienst-

leistungen der Bibliothek in Anspruch zu nehmen. Für die

leistungen der Bibliothek in Anspruch zu nehmen. Für die
Netzverbindungen im regionalen und im überregionalen

Netzverbindungen im regionalen und im überregionalen
Bereich sind daher zusätzliche Kosten der Installation und des Betriebes zu veranschlagen.

Bei der Begutachtung des hessischen HBFG-Antrages zur Finanzierung der PICA-Migration kamen obige DFG-Grundsätze voll zum Tragen. Dies bedeutete allerdings, daß in Hessen nicht das im Norddeutschen Bibliotheksverbund bereits eingesetzte und erprobte PICA auf VMS-Basis eingesetzt werden konnte, sondern ein PICA-System auf UNIXBasis (DEC-UNIX) zu etablieren war, das in dieser Form damals noch nicht vollständig existierte.

Gegenüber der norddeutschen DV-Organisation (Gemeinsamer Bibliotheks-Verbund - GBV), die mit dem Bibliotheksrechenzentrum Niedersachsen (BRZN) in Göttingen über eine zentrale Bibliotheks-DV verfügt, verfolgte man in Hessen einen dezentralen Weg, der den einzelnen Bibliotheken resp. Hochschulen größeres Gewicht und Verantwortung zuteil werden ließ. Der hessische PICA-Zentralrechner (CBS) wird von einer DV-Einheit (BDV), die dem Frankfurter Hochschulrechenzentrum (HRZ) zugeordnet ist, betrieben. An den einzelnen Hochschulstandorten wurden Lokalsysteme (LBS) (Server und PC's) eingerichtet, die an den meisten Standorten vom jeweiligen $R Z$ betreut werden.

In Kassel obliegt die Administration des LBS und der Datenbanken sowie der gesamte PC-Einsatz der Bibliothek; die reine Hardware- und Betriebssystem-Betreuung der drei PICA-Server wird vom HRZ Kassel übernommen. Die vom HRZ bereitgestellte Netzinfrastruktur ist integrierendes Element des Systems.

Ab dem 1.10.1995 war der PICA-Server in Kassel mittels Telnet-Verbindung von jedem Rechner mit TCP/IP-Zugang zugänglich und recherchierbar. Die zeitaufwendige Suche mit Microfiche-Lesegeräten entfiel damit weitgehend. Mitte 1997 wurde der Telnet-Zugriff auf den OPAC um einen zeitgemäBen WebOPAC ergänzt.

Über ein frei verfügbares Auswertungstool (Webalizer) lassen sich aus den WWW-Server-Statistiken auf dem Web Grafiken und Auswertungstabellen produzieren, auf denen klar die rasch steigende Akzeptanz des WebOPAC abzulesen ist. 


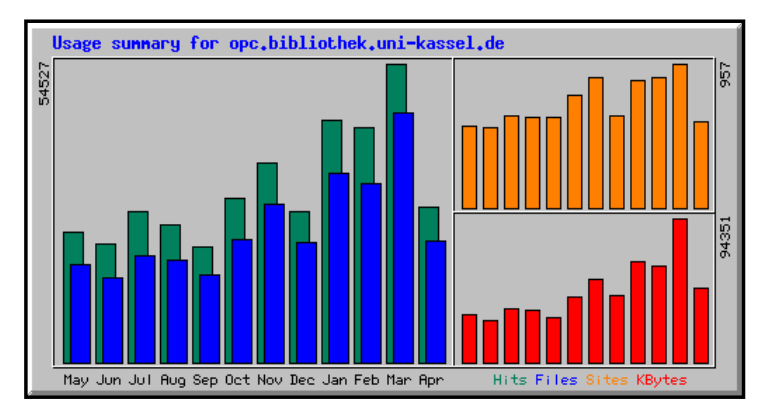

Abb. 5: Jahresstatistik des WebOPAC im Internet (Stand 15.4.99)

Wie in anderen Auswertungsprogrammen von WWW-Statistiken ist u.a. auch eine Auswertung der Nutzung über den gesamten Tag möglich. Erwartungsgemäß spiegeln sich hier, beispielhaft verdeutlicht an der Auswertung der Zahlen für März 1999 (Semesterferienmonat), deutlich die Nutzungsgewohnheiten der WebOPAC-Befrager über eine am Morgen von 7.00 bis 10.00 Uhr stark ansteigende Nutzung, gefolgt von einem um die Mittagszeit ( 10.00 bis $14.00 \mathrm{Uhr}$ ) fast gleichbleibenden Höchststand (kleiner Einschnitt um 12.00 Uhr: Mittagessen), um schließlich in der Zeit von 15.00 bis 18.00 Uhr wieder ebenso schnell abzufallen. Der Nutzungslevel, der etwa um 8.00 Uhr morgens erreicht wurde, hält sich auf dieser Höhe von 18.00 Uhr fast gleichbleibend bis $23.00 \mathrm{Uhr}$.

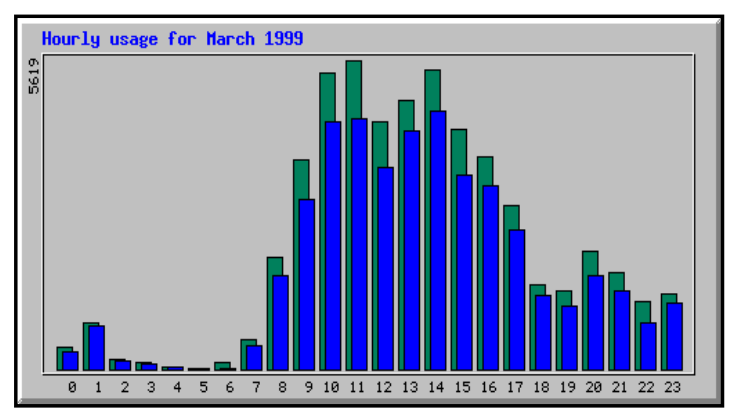

Abb. 6: Statistik: Stündliche Nutzung Web-OPAC März 199؟

Pilotanwender. Neben der routinemäßigen Software-Pflege des PICA-Systems und der Installation neuer PICA-Module (z.B. Fernleihe 1998/99) fungierte die Gesamthochschulbibliothek auch als Pilotanwender. Im Rahmen der dezentralen PICA-Lokalsysteme (LBS) hat es die Gesamthochschulbibliothek Kassel zweimal übernommen, Pilotanwender bei der Einführung von PICA-Modulen zu sein und beteiligte sich zusätzlich bei der Einführung des Fernleih-Moduls:

- In den Jahren1996/97 erfolgte die Organisation und

Durchführung der Übernahme von Medien-, Leser- und Ausleihdaten aus dem Vorgängersystem BIBDIA nach PICA-OWC bzw. PICA-OUS (Ausleihe) als hessische Pilotbibliothek in Zusammenarbeit mit der FH GießenFriedberg und der StUB Frankfurt/M.

- In den folgenden Jahren 1997/98 erfolgte die Organisation und Durchführung eines Testsystems ACQ (Erwerbung) für die Erwerbung der StUB Frankfurt/M sowie anderer Bibliotheken in Hessen. Dies führte zu einer Zusammenarbeit mit der StUB bei deren Einführung von PICA-ACQ für die Monographienerwerbung und einer Schulung der Parametrisierung von PICA-ACQ für Kollegen/innen der hessischen Bibliotheken durch die GHB.

\section{Bibliotheks-PC-Netz}

Im Hauptgebäude und in den Bereichsbibliotheken wurden Mitarbeiter- und Benutzer-PC's unter Windows und Windows NT installiert, die neben einem Online-Zugriff auf PICA (OPAC) auch eine kostenlose Benutzung des neuen CD-
ROM-Netzes vorsehen. Mittels eines gültigen Accounts bzw. eines auf das Wissenschaftsnetz beschränkten Gastzuganges können von diesen Rechnern zudem Internet-Recherchen gestartet werden.

Auf den jeweiligen Benutzer-PC's (OPAC-PC's) wurde Windows NT Workstation installiert, um trotz umfangreicher Arbeitsmöglichkeiten dennoch ein hohes Maß an NetzwerkSicherheit gewährleisten zu können. Zur Diebstahlsicherung wurden die Computergehäuse mit Schlössern versehen, Maus und Tastatur über ein weiteres Steckschloß gesichert. Bei der Anmeldung der Mitarbeiterrechner ins Bibliotheksnetz erfolgt in der Regel gleichzeitig auch die Anmeldung auf dem POP-Mailer der Bibliothek, so daß die elektronische Post des Vortages sofort sichtbar wird. Jeder Bibliotheksmitarbeiter, der über einen PC verfügt, besitzt gleichzeitig eine E-Mail-Adresse in der Sub-Domain "bibliothek.uni-kassel.de". Durch das Anmelden auf dem NT-Server werden jedem Mitarbeiter zudem die Disk- und Druckdienste des Servers zugeordnet. So können die Mitarbeiter große Druckjobs auf den vorhandenen Abteilungsdruckern (24 S/min PS) ausdrucken, sofern sie sich einen anderen Drucker als den standardmäßig eingestellten Arbeitsplatz- oder Arbeitsgruppen-Drucker zuordnen. Durch das Abspeichern wichtiger Dokumente auf den Benutzerpartitionen des NT-Servers kann jeder Mitarbeiter durch den laufenden Backup-Service des Servers selbst für eine gewisse Datensicherheit sorgen.

\section{Schulungsmaßnahmen}

Bei Einführung der PICA-Softwaremodule wurde frühzeitig erkannt, daß umfangreiche Schulungen vonnöten waren, die bislang nicht in DV-Problemen geschulten Mitarbeiter der Bibliothek mit diesem für sie i.d.R. neuen Medium vertraut zu machen. Daher wurde aus Eigenmitteln der Bibliothek ein Schulungsraum mit 9 PC's und einem zentralen Laserdrukker eingerichtet. Auf den Rechnern befinden sich drei Betriebssysteme (Windows 3.11, Windows NT und Linux), so daß wahlweise das gewünschte Betriebsystem ausgewählt werden kann. Dieser Schulungsraum wird nicht nur zu reinen DV-Schulungszwecken benutzt, sondern hier finden auch interne Weiterbildungsveranstaltungen anderer Abteilungen der Bibliothek statt.

Von der Abt. I'M wurden in den Jahren 1995-98 folgende interne Bibliotheks-Kurse angeboten:

\section{Kurstitel}

Der Bibliotheks-EDV-Arbeitsplatz Einführung in die CD-ROM WISO Nutzung des Internet

Benutzung der CD-ROM-Anlage

Wie funktioniert mein PC?

Windows für Anfänger

Wie kann ich FTP nutzen?

Die Bibliothek als IntraNet

Einführung in E-Mail

WinWord 6.0 für Anfänger

WinWord 6.0 für Fortgeschrittene

Allg. PC-Grundkenntnisse

Nutzung von CD-ROM-

Datenbanken unter Windows

Erstellen von HTML-Dokumenten

Excel

Erstellen von WWW-Dokumenten Win-IBW

Informationsquellen im Internet

Betriebssystem Windows NT 4.0

Summe 1995-98

\begin{tabular}{|c|c|c|}
\hline 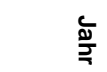 & 蛋 $\frac{\bar{c}}{\bar{c}}$ & 号㐫 \\
\hline 1995 & 4 & $\theta$ \\
\hline 1995 & 4 & \\
\hline $1995-96$ & 2 & 2 \\
\hline 1995-96 & 2 & 3 \\
\hline 1996 & 1 & \\
\hline 1996 & 5 & \\
\hline 1996 & 1 & \\
\hline 1996 & 1 & \\
\hline $1996-98$ & 11 & \\
\hline 1997 & 3 & \\
\hline 1997 & 1 & \\
\hline 1997 & 1 & \\
\hline 1997 & 3 & \\
\hline 1997 & 4 & \\
\hline $1997-98$ & 3 & \\
\hline 1998 & 2 & \\
\hline 1998 & 3 & \\
\hline 1998 & 1 & \\
\hline 1998 & 9 & \\
\hline & 61 & \\
\hline
\end{tabular}


Deutlich erkennbar ist innerhalb der Jahre das parallel zum DV-Ausbau bestehende Interesse der Mitarbeiter der Bibliothek, sich entsprechend fortzubilden. Während am Beginn der Schulungszeit 1995 die Kurse über hohe Teilnehmerzahlen pro Kurs verfügten, um der schnell vonstatten gehenden PICA-Migration eine Basis zu schaffen, wurden ab 1996 die Kursgrößen an die Anzahl der zur Verfügung stehenden PC's angepaßt.

\section{Verbesserungen im bibliothekari- schen Alltag}

\section{IntraNet}

Im Rahmen der Neugestaltung des WWW-Angebotes bot sich die Gelegenheit, die IP-Dienste der Mitarbeiter-PC's gleichzeitig für ein Intranet einzusetzen. Hierzu wurde auf dem NT-Server eine Partition eingerichtet, die in HTML-Format die bibliotheksinternen Nachrichten, Bekanntmachungen und Protokolle enthält. Durch das Einloggen der Mitarbeiter in das bibliothekseigene NT-Netz wird ihnen diese Partition automatisch zugelinkt, so daß sie über den WWW-Client auf diese Dokumente zugreifen können. Auf diese Weise ist zudem sichergestellt, daß kein Außenstehender an diese Informationen gelangen kann. Mittlerweile nutzen auch bereits einzelne Arbeitsbereiche und Bereichsbibliotheken diese Möglichkeiten der internen Kommunikation, indem beispielsweise in Intranet die Schichtpläne für die Spätdienste stets auf dem aktuellsten Stand einsehbar sind.

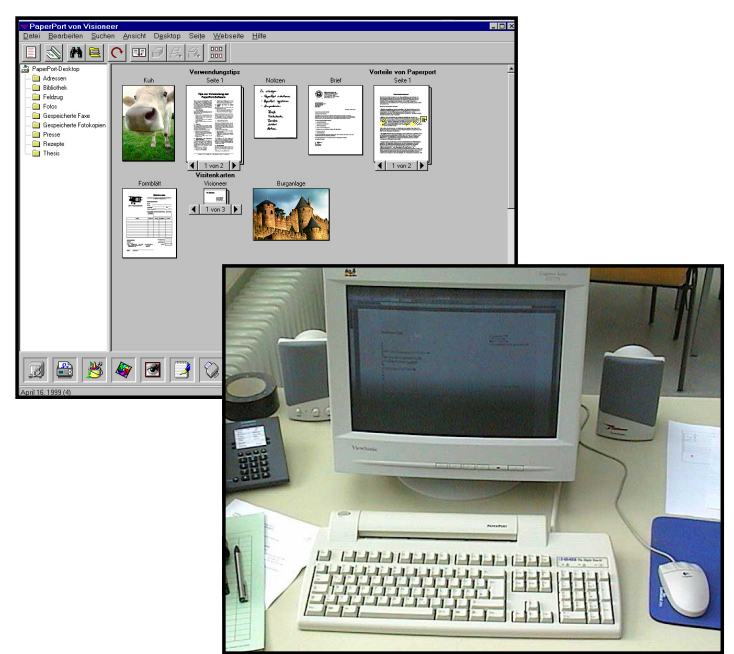

Abb. 7: Paperport-Arbeitsplatz und Paperport-Bildschirm

Aufgrund der Dezentralisierung auch am Hauptstandort werden die dortigen Bereichsbibliotheken verstärkt neue Kommunikationswege benötigen. $\mathrm{Da}$ aus Kostengründen (Steckkarte UPO-S0, a/b-Wandler, Faxgerät) die Anschaffung weiterer Faxgeräte zunächst zurückgestellt wurde, wurde ein Pilotprojekt zwischen Ausleihe, der Bereichsbibliothek $\mathrm{CO}$ und der Abt. I'M durchgeführt, bei dem der Einsatz der EMail für Geschäftsgangsprozesse untersucht wurde. Über angeschlossene kleine Durchzugsscanner (Paperport) an der Tastatur können auch Papiervorlagen schnell gescannt, archiviert bzw. per Drag-and-Drop auf ein E-Mail-Button gezogen werden. Dadurch wird die E-Mail-Software gestartet und automatisch das gescannte Bild als sog. "Attachment" angehängt. Die gesendete Mail kann beim Adressaten gelesen und das gescannte Bild automatisch in einem Viewer angesehen und eventuell gedruckt werden. Derzeit werden alle Bereichsbibliotheken mit Paperport-Tastaturen ausgestattet. Sofern man die interne Kommunikation ausbaut, besteht konsequent weitergedacht der nächste Schritt darin, darauf aufbauend auch die externe Kommunikation anzupassen.
Daher wurde seitens der Abt. I'M unter LINUX ein Fax-Server installiert und administriert, der derzeit intern Faxe im Hochschulnetz von jedem Mitarbeiterarbeitsplatz technisch zuläßt und auch, bei entsprechender Berechtigung, Faxe nach auBerhalb ermöglicht. Nachdem es nunmehr möglich war, auch eine zweistellige zentrale Faxnummer zu erhalten, wird in absehbarer Zeit auch der umgekehrte Weg, nämlich der Empfang von Faxen und das Weiterleiten an den betreffenden Mitarbeiter, realisierbar sein. Benutzt wurde eine frei verfügbare Faxserversoftware namens HylaFAX.

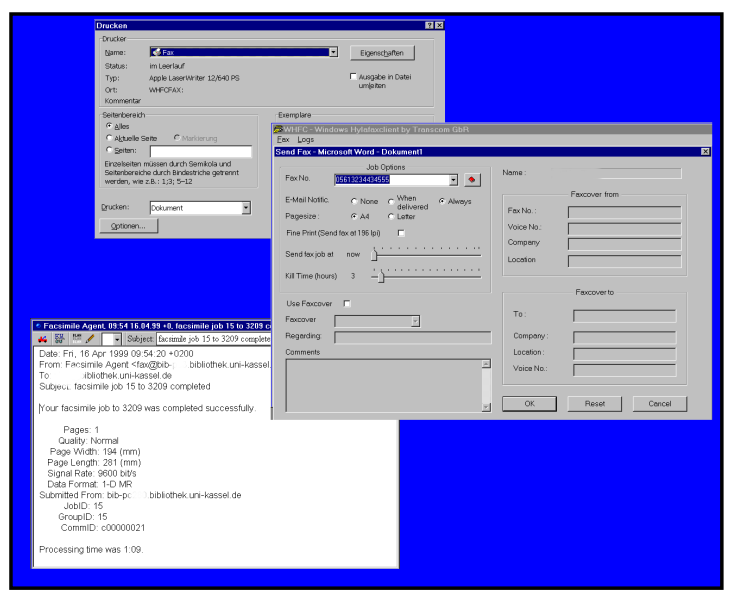

Abb. 8: Fax-Server

Entsprechende Clientsoftware liegt für diverse UNIX-Derivate, MAC's und NT-Rechner vor.

\section{Ausleiharbeitsplatz}

In Zusammenhang mit der zukünftigen Einführung von Ausleiharbeitsplätzen, die über grafische Oberflächen (z.B. Windows) verfügen, hat sich die Abt. I'M Gedanken über die pragmatische Umsetzung dieser Arbeitsplätze unter den gegebenen Umständen Gedanken gemacht.

Bislang bestehen die Ausleiharbeitsplätze aus einem DOSRechner mit einem entsprechenden PICA-Softwaremodul (DOS-IBW), wobei über eine Y-Verkabelung an der Tastatur ein sogenannter Kombileser für die beiden an der GHB vorhandenen Buchetiketten (Nixdorf-Barcode, OCR-B) angeschlossen ist. Über einen an der Ausleihtheke angebrachten Magneten werden die auszuleihenden oder zurückzugebenen Bücher magnetisiert bzw. entmagnetisiert. Geht man nunmehr von einer grafischen Oberfläche aus, so ergibt sich zwangsläufig durch die Bedienung dieser Oberfläche (z.B. mittels einer Maus) ein weiteres Gerät auf der Ausleihtheke, was letztlich einen permanenten Wechsel von Geräten während eines Ausleih- oder Rückbuchungsvorganges bedeutet.

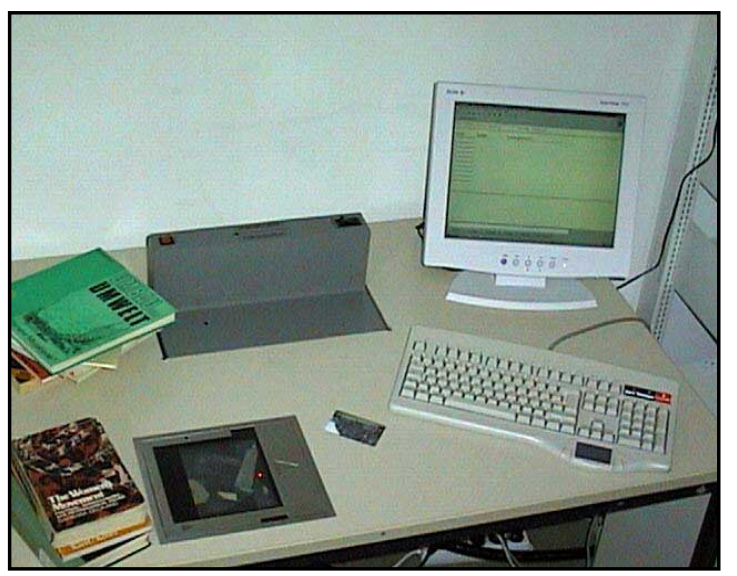

Abb. 9: Prototyp Ausleiharbeitsplatz 
Daher wurde Ende 1998 ein Prototyp eines Ausleiharbeitsplatzes mit grafischer Oberfläche entwickelt, der vorsieht:

- Statt des bisherigen Kombilesers oder (nach Umstellung auf Barcode) eines reinen Hand-Barcode-Scanners wird ein Tisch-Scanner eingesetzt, der bündig mit der Tischoberfläche in den Ausleihtresen eingelassen ist. Dieser verfügt zudem über ein größeres Erkennfeld.

- Für die Bedienung der grafischen Oberfläche wird statt einer Maus eine Tastatur mit Trackpad eingesetzt.

- Als De- bzw. Aktiviereinheit wird ein Magnetisierungskomplex eingesetzt, der softwaremäßig über ein Protokoll mit der PICA-Software kommunizieren kann.

- Um die Problematik der elektromagnetischen Störfelder zu minimieren, sind 15"-TFT-Displays als Monitore vorgesehen, die in etwa dem Sehfeld eines herkömmlichen 17"-Monitors entsprechen.

Bis zum endgültigen Einsatz dieser Ausleiharbeitsplätze sind seitens der betreffenden Mitarbeiter Überlegungen zur endgültigen Anordnung der Bestandteile im Sinne eines effektiven Arbeitsablaufes zu treffen.

\section{Umetikettierung}

In der fast 25jährigen Geschichte der GHB wurden zwe wichtige Entscheidungen bei den Buchetiketten getroffen. Anfänglich benutzte man in Kassel einen alten Nixdorf-Barcode, später wechselte man zu OCR-B-Etiketten. Da beide Etiketten nebeneinander in Benutzung sind, mußte notgedrungen die Leseautomatik so erworben werden, daß beide grundsätzlich unterschiedlichen (Strichcode und Klarschrift) Systeme lesbar waren. Diese Problematik hat nicht unerhebliche finanzielle Folgen bei der Beschaffung dieser Leseautomatiken. Während eine Standard-OCR-B-Lesepistole ca. 1.500 ,- bis 2.000 ,- kostet, schlägt eine Kombilesepistole mit ca. 4.500,- bis 6.000,- DM zu Buche. Da die Leseleistung (abhängig von der Optik) im Laufe der Jahre abnimmt, muß mit Wiederbeschaffungszyklen von ca. 3-4 Jahren gerechnet werden.

Da zudem die Kombileser sehr anfällig sind, sich also häufig in der Reparatur befinden, galt es, eine Lösung dieses Problems zu finden. Erschwerend kam hinzu, daß die bislang selbst hergestellten OCR-B-Etiketten nach Ablösung des BIBDIA-Rechners extern kommerziell geordert werden mußten. Einer der wenigen verbliebenen Anbieter für diese Etiketten konnte nur zögerlich und qualitativ nicht immer befriedigend diese kaum noch gebräuchlichen Etiketten liefern.

Die GHB entschloß sich daher auch in Anbetracht der SBTerminal-Frage, die zwingend einen Barcode voraussetzte, ab Mitte 1997 ausschließlich Barcode-Etiketten für Neuanschaffungen zu verwenden. Anschließend wurde mit einem Pilotversuch begonnen, die Bestände zweier Bereichsbibliotheken $(A 0, C 0)$ rückwirkend mit diesen Etiketten zu versehen.

Ziel ist es, zumindest am Hauptstandort die Buchetiketten auf einen gemeinsamen Stand zu bringen. Für diese Retroklebeaktion stellte die Abt. I'M ein Set von Lesepistole, Rechner mit Etikettierungssoftware und Etikettendrucker zusammen, an dem unter bibliothekarischer Aufsicht studentische Hilfskräfte ältere Bücher zusätzlich mit dem neuen Barcode versehen. Die neuen Barcode-Etiketten werden von der bisher eingesetzten Kombilesepistole hervorragend gelesen, ein demnächst anzuschaffender reiner Barcodeleser kostet zudem nur 500,- bis 1000,- DM. Zum gegenwärtigen Stand sind am Hauptstandort drei der insgesamt fünf Bereichsbibliotheken fast vollständig auf den neuen Barcode umgestellt.

Nach einer umfassenden Kostenanalyse durch die Abteilung wurde in Kassel beschlossen, die Buchetiketten selbst herzustellen. Nach Prüfung der Klebematerialien, Papier resp.

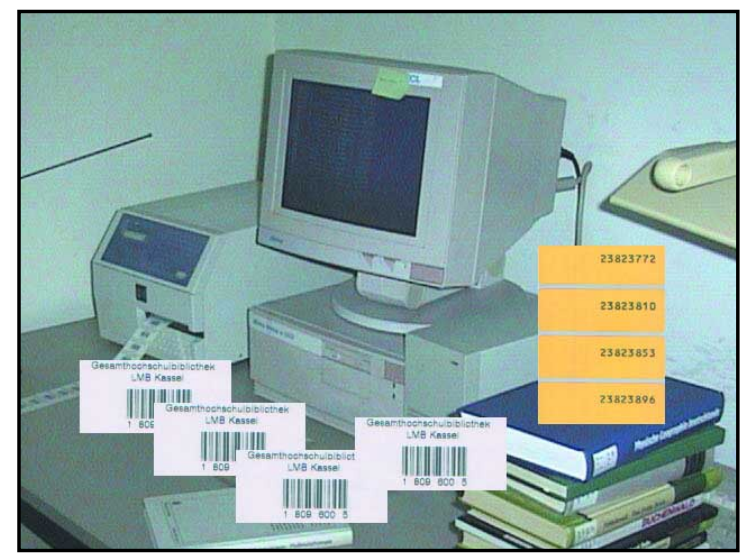

Abb. 10: Etikettierstation mit alten OCR-B- und BarcodeEtiketten

PVC-Qualitäten und Programmierung eines Druckprogrammes werden nunmehr seit knapp eineinhalb Jahren ohne Probleme diese Etiketten gedruckt und angewandt.

\section{Initiierung und Durchführung von er- weiterten Dienstleistungsangeboten}

\section{Selbstverbuchungsterminals}

In der Gesamthochschulbibliothek wurden im Rahmen der Erweiterung des Dienstleistungsangebotes, vier sogenannte Selbstverbuchungsterminals für Benutzer installiert. Diese Selbstverbuchungsplätze bestehen aus:

- einer Steuerelektronik, die mit dem Ausleihsystem verbunden ist

- einem Kartenlesegerät für die Identifikation des Lesers (Barcode)

- einem Medienlesegerät für die Identifikation des zu verleihenden oder zurückzunehmenden Buches (Barcode)

- Tastatur und Bildschirm als Benutzer-Schnittstelle

Mit diesen Systemen können die Bibliotheksbenutzer Bücher, die mit dem neuen Barcode-System ausgestattet sind, selbständig ausleihen. Die auszuleihenden Bücher sind für

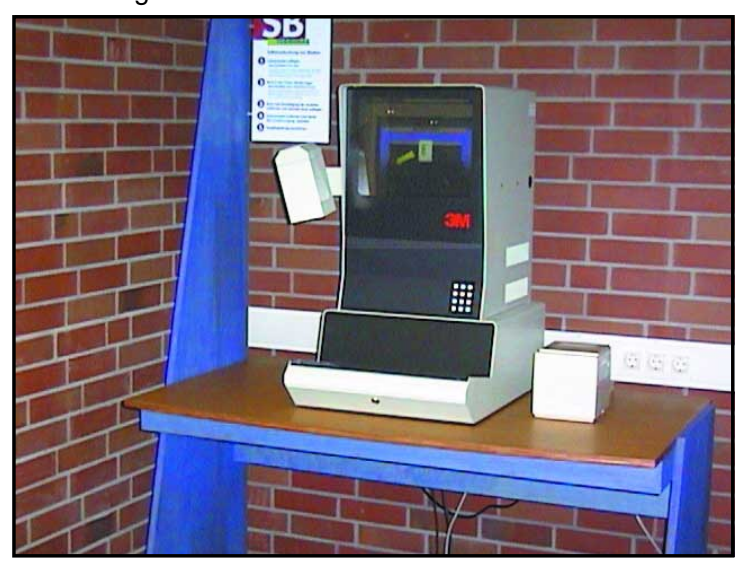

Abb. 11: SB-Terminal am Eingang des Hauptgebäudes

Benutzer dadurch gekennzeichnet, daß sich das Verbuchungsetikett auf der Rückseite des Bucheinbandes befindet. Bei der jeweiligen Transaktion wird der Magnetstreifen entsprechend magnetisiert, so daß keine unberechtigten Entleihungen stattfinden können. Wie bei einem Geldscheinautomaten erfolgt eine leicht verständliche Bildschirmführung über einen Monitor. Das System ist voll in das PICAAusleih-Modul integrierbar. Derzeit ist nur eine Ausleihe an diesen Terminals geplant. Es ist jedoch technisch möglich, in mittelfristiger Zeit mit diesen Terminals auch die Rücknahme 
von Büchern zu realisieren. Um eine reibungslose Funktion dieser Selbstverbuchungsterminals zu gewährleisten, mußten zudem Überlegungen angestellt werden, wie zukünftig Leserausweise und Buchetikettierung zu gestalten sind (s. Barcode oben).

Im Eingangsbereich wurde eines der SB-Terminals auf einen speziell angefertigten Tisch, der rechts und links genügend Platz für das Abarbeiten mehrerer Bücher bietet, gestellt. Im Rahmen der Beschaffung ist gleichsam die bisherige statische und wenig funktionale Buchsicherungsanlage durch eine neuere, leichter zu verankernde Anlage ersetzt worden. Gleichwohl verbunden mit der Buchetiketten- und SB-Terminal-Frage waren die Leserausweise. Da ohnehin Nachbestellungen bevorstanden, wurde 1997 ein Leserausweis gestaltet, der neben einer Darstellung der GHB auch einen zusätzlichen Barcode trägt. Nur mittels dieses Leserauswei-

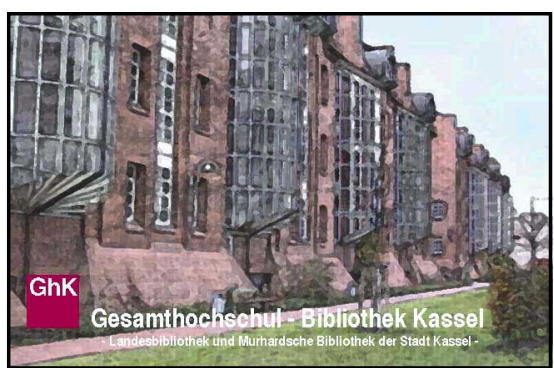

Abb. 12: Vorderseite des Leseausweises

ses ist die Verbuchung auf den SB-Terminals möglich. Bei der Beschaffung der Terminals wurde als Option u.a. vereinbart, daß an diesen SB-Verbuchungsgeräten optional ein Chipkartenlesegerät als Benutzererkennung betrieben werden kann, um sicherzustellen, daß die an der GHK in Aussicht gestellten Chipkarten für Studenten dann auch in diesem Bereich benutzbar sind. Über diese Chipkarte wären allerdings nicht alle Benutzer der Bibliothek abgedeckt. Da die GHB gleichzeitig Aufgaben einer Landesbibliothek wahrnimmt, mußte sichergestellt werden, daß die wissenschaftlichen Bediensteten und nicht GHK-interne Benutzer diese Dienstleistung der Bibliothek ebenfalls nutzen können.

Die GHB verfügt derzeit über vier derartige SB-Terminals im Hauptgebäude, die insbesondere in der Zeit von 18.00 . 21.00 Uhr, wenn die Ausleihe nicht mehr besetzt ist, eifrig genutzt werden. Die Bedingungen für die Aufstellung von Selbstverbuchungsterminals waren in Kassel besonders günstig, da die GHB keine Magazinbibliothek ist, sondern ihre Bestände frei zugänglich aufstellen konnte.

\section{Multi-Media-Thek (MMT)}

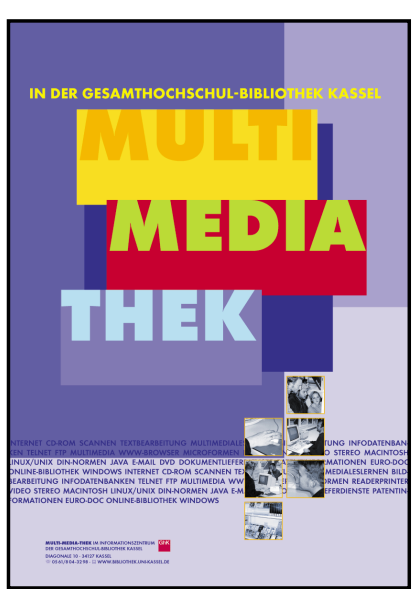

Abb. 13: Info-Poster zur MMT
Im Informationszeitalter erhalten die Neuen Medien eine immer größer werdende Bedeutung. Lange Zeit galten gedruckte Informationen häufig in Form von Büchern und Zeitschriften - als eigentliche Informationsquelle in den $\mathrm{Bi}$ bliotheken. Mehr und mehr nehmen jedoch mittlerweile auch multimediale Anwendungen breiten Raum ein, gilt es doch, sich den veränderten Lese- und Informationsgewohnheiten anzupassen. Insbeson- dere sollen elektronische Informationsquellen und digitale Literatur erschlossen und bereitgestellt werden.

Im Zuge dieser Entwicklung wurde durch die hessische Ministerin am 11.11.1998 in der Gesamthochschulbibliothek Kassel eine neugeschaffene Multi-Media-Thek (MMT) eröffnet. In dieser Multimediathek sollen die Bibliotheksbenutzer

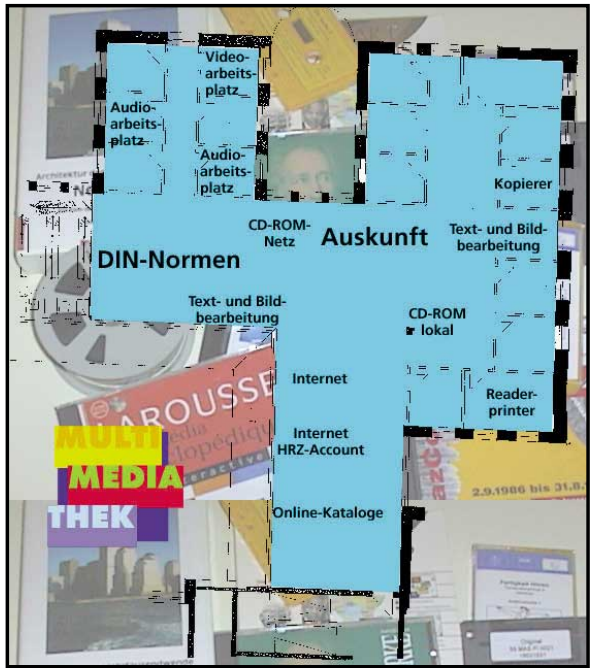

Abb. 14: Überblicksplan Multi-Media-Thek

die Möglichkeit erhalten, die Neuen Medien zu erkunden und gewinnbringend für ihre Fragestellungen einzusetzen. Neben den mittlerweile weiter verbreiteten Internetarbeitsplätzen haben die Benutzer Gelegenheit, an speziell ausgestatteten Arbeitsplätzen Audio- und Videoanwendungen zu testen. Die zur Verfügung stehenden Arbeitsplätze sind plattformübergreifend gestaltet, neben Windows NTRechnern können Macintosh-, Java- und Linux-Rechner benutzt werden.

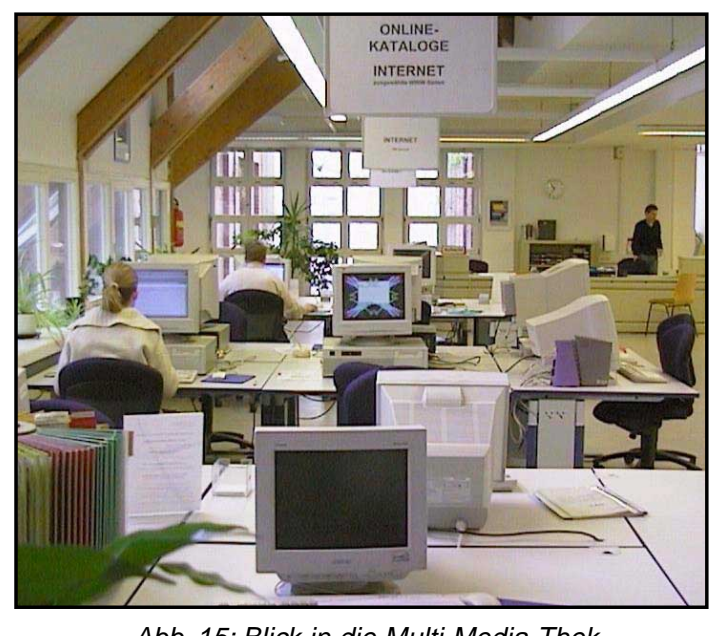

Abb. 15: Blick in die Multi-Media-Thek

Alle PC-Systeme verfügen über hochauflösende Grafikkarten und Soundkarten, allerdings müssen zum Abhören von Audio-Medien, DVD's oder Multimedia-CD's entsprechende Kopfhörer aufgesetzt werden. Über WinCenter können auch auf Linux- und Macintosh-Rechnern CD-Applikationen aus dem GHB-internen CD-Server angewählt werden. Zur Auswahl stehen derzeit 140 Datenbanken, die zudem ergänzt werden durch entsprechende Datenbankzugriffe auf den Silverplatter-Server der GHB. In separaten Klein-Arbeitsräumen können Audio-Cassetten und Video-Bänder benutzt werden.

Natürlich lassen sich auch herkömmliche Dias und Microfiches in den Räumen der MMT betrachten und Ausdrucke 
über den Reader-Printer bzw. über das Bibliotheksnetz auf einen zentralen Benutzer-Drucker (OCE-Drucksystem mit Entgeldregelung) machen. Als Auslegestelle für DIN-Normen kann im DIN-Katalog über Internet recherchiert und das Ergebnis der Recherche direkt im DIN-Bestand angesehen werden.

In einem speziell eingerichteten Macintosh-Pool sind mittels Scanner, Grafikbearbeitungssoftware und OCR-Möglichkeit weitere Bedingungen für multimediales Arbeit geschaffen.

\section{Kostenpflichtige Druckstation für Benutzer}

Im Rahmen der damaligen Überlegungen zur Gestaltung der Benutzer-Arbeitsplatze hatte die Abt. I'M die Idee, innerhalb der NT-Domäne neben zentralen Abteilungsdruckern auch eine zentrale Druckmöglichkeit für die Benutzerarbeitsplätze einzurichten. Die Realisierung nahm einen längeren Zeitraum als eigentlich geplant ein, da die Bedingungen, daß jeder Benutzer aus Sicherheitsgründen seinen Druck-Job mit einem Passwort versehen muß und weiterhin gewährleistet sein soll, daß diese Ausdrucke kostenpflichtig sein sollen, in der Anfangszeit von Windows NT 1995/6 nur schwerlich einem Hersteller nahezubringen waren. Seit Beginn 1998 jedoch waren die Bemühungen und Verhandlungen erfolgreich, da über einen Rahmenvertrag der GHK ein Hersteller gewonnen werden konnte, der bereit war, eine digitale Kopier- und Druckstation zentral im Hauptgebäude der Bibliothek aufzustellen und wartungsmäßig an die GHB-NTDomäne anzuschließen. Seitdem kann jeder Benutzer seine Ausdrucke über die Benutzer-PC's an diesen Drucker senden. Der Druck erfolgt technisch über das Ipr-Protokoll zu einem unter OS/2 betriebenen Druck-Spooler, der der Druckstation vorgeschaltet ist.

Beim Drucken aus den Applikationen der Benutzer-PC's heraus erfolgt nach Bestätigung des Druckauftrages ein Dialog mit Druckoptionen, der die Einstellung zusätzlicher Druckparameter (Anzahl der Kopien, PIN-Code, Bindung, Duplex, Bildqualität, Heften) steuert. Der Drucker ist voreingestellt, der PIN-Code muß zwingend eingegeben werden.

Sobald der Druck-Job abgeschickt wurde, kann der Nutzer an dem zentral aufgestellten Drucker mittels eines LCD-Displays seinen Druckauftrag anwählen und inn mittels Eingabe seines vergebenen PIN-Codes (sechsstelliger Zahlencode) entsperren und zum Druck freigeben. Der Druck erfolgt, wenn eine Bezahlung erfolgt. Wie beim Kopieren kann die Bezahlung durch eine wiederaufladbare Kunstoff-Checkkarte (GHK-Standard) oder Bargeld erfolgen.

\section{InterNet-PC's}

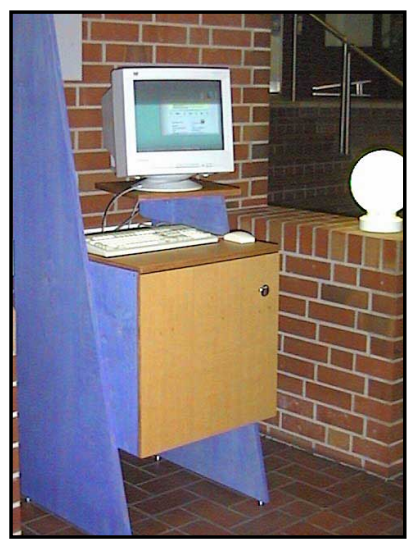

Abb. 16: Internet-PC am Eingang des Hauptgebäudes

rechtliche und organisatorische Probleme, da diese PC's zumindest in der Zeit ab 18.00 Uhr nicht mehr beaufsichtigt werden könnten. Daher wurden zunächst drei Internetzugänge unterschieden:

- Anonymer Internetzugang auf einem PC. Der Nutzer muß sich bei der Aufsicht ausweisen und erhält (gegebenenfalls zeitlich limitierten) kostenlosen Zugang zum Internet. Diese PC's stehen in unmittelbarer Sichtweite der Aufsicht.

- Anonymer Internetzugang auf alle dem WIN (Wissenschaftsnetz) angeschlossenen Institutionen.

- Internetaccount des Hochschulrechenzentrums (HRZ). Der Nutzer unterliegt den Nutzungsbestimmungen des HRZ.

Die Abt. I'M hat zur Realisierung dieses Internet-Konzepts die frei zugänglichen Rechner nur in der Multi-Media-Thek aufgestellt, wo eine entsprechende Aufsicht gewährleistet ist. Die Benutzer-PC's in den Bereichsbibliotheken der GHB verfügen dagegen wahlweise auf LINUX-Basis über die o.a. letzten beiden Zugangsmöglichkeiten. Der anonyme auf das WIN beschränkte WWW-Zugriff wird technisch durch einen Squid-Server realisiert, über den die Zugriffsbestimmungen definiert werden können.

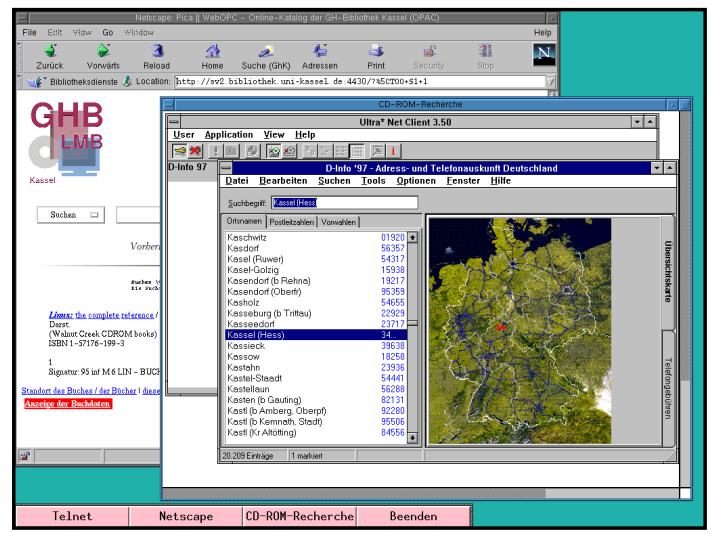

$A b b$. 17: Oberfläche derlnternet- $P C$

Beim Starten der Rechner kann entweder der Zugang zu HRZ-Rechnern oder der anonyme Zugang ausgewählt werden. Bei der Wahl des Gast-Zuganges wird dem Benutzer automatisch am unteren Rand des Bildschirms ein Menü mit den Punkten:

Telnet

Netscape

CD-ROM

Beenden

angeboten. Über den ersten Menüpunkt ist der Telnet-Zugang zu jedem Rechner möglich, z.B. zum PICA-OPAC. Der Punkt „Netscape“ ermöglicht die Nutzung der WWW-Angebote innerhalb des WWW. Rechner außerhalb des DFN (Deutschen Forschungsnetzes) können nicht angesprochen werden. Aus Sicherheitsgründen wurden zudem einige Funktionen des Netscape-Browsers gestrichen, so ist es u.a. nicht möglich, abzuspeichern, E-Mails zu versenden, Einstellungen des Browsers zu verändern oder Bookmarks zu setzen. Der Druck von WWW-Seiten ist über den zentralen kostenpflichtigen Druckserver sofort möglich. Die Auswahl "CD-ROM“ startet den Zugriff mittels WinCenter auf die Datenbanken des UltraNet-CD-Servers. Hierbei kann der Nutzer seine Rechercheergebnisse zwar nicht abspeichen, jedoch über die o.a. OCE-Druckstation ausdrucken.

\section{Campusweite CD-Server}

Im derzeit installierten CD-ROM-Server (UltraNet) sind die CDs nicht mehr in CD-Türmen zugreifbar, sondern werden komplett als Imagedateien auf Festplatten kopiert. Daher ist der Zugriff erheblich schneller und von mehreren Benutzern 
gleichzeitig möglich. Als Rechnerplattform dient ein NT-Server.

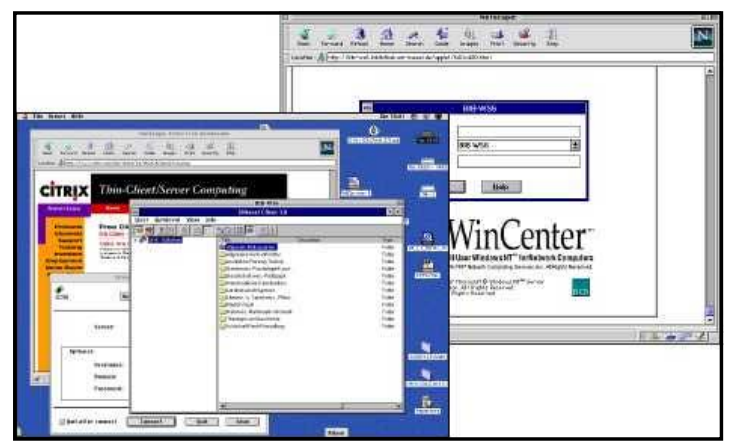

Abb. 18: CD-ROM-Server mittels WinCenter auf dem Mac

Im Jahre 1997 konnten über zusätzliche Drittmittel die Anzahl der Datenbanken auf dem UltraNet und SilverplatterServern erheblich ausgeweitet werden, so daß der Plattenplatz dieser Server ergänzt werden mußte. Zur Absicherung der Dateninhalte wurde 1998 ein Raid-Array $\left(11^{*} 9\right.$ Gbyte, Raid Level 5) an den UltraNet-Server angeschlossen.

Die Nutzung dieser Anlage konnte campusweit seit 1995 von allen am Hochschulnetz angeschlossenen PC's erfolgen. Entsprechende Client-Software ist kostenlos über die GHB erhältlich. Anfänglich (Ende 1996) waren über drei sogenannte Dual-OmniWare-PC's die DOS-Retrieval auch für UNIX- und Macintosh-Rechner über X-11-Technologie verfügbar. Dieser Zugriff war allerdings nicht besonders komfortabel und naturgemäß für Windows-Retrieval nicht einsetzbar. Um diesem Mißstand abzuhelfen, wurde Ende 1997 sowohl die UltraNet-Installation auf ein neues Release mit verbesserter Unterstützung von Windows-Retrieval umgestellt als auch ein WinFrame-Server der Firma Citrix aufgesetzt. Die bisherigen Ergebnisse sind sehr positiv. Die zu Beginn angeschaffte 15-Lizenz wurde rasch aufgestockt, da der Zugriff über diese ICA-Clients zum Applikationsserver stark zugenommen hat.

Auf dem SUN-Cluster der Bibliothek konnte neben dem für das Projekt WebDOC notwendigen Web-Server mit PICAGoldenURL-Zugang 1996 auch ein Silverplatter-Server installiert werden. Einige CD-Bestände wurden bereits gleich zu Beginn vom UltraNet-Server auf diesen Server umgesetzt (z.B. Medline), da sich der mit einem üblichen Webbrowser mögliche Zugang zu diesem Client-Server-basierten Datenbanksystem als sehr einfach gestaltet.

Da die Resonanz der Benutzerschaft insbesondere wegen der durchgehend einheitlichen WWW-Abfragestruktur bei allen angebotenen Datenbeständen auf diese Webzugangsmöglichkeit sehr positiv war, wurden bei den nachfolgenden Beschaffungen darauf geachtet, bei gleichen Angeboten hinsichtlich Umfang und Preis möglichst die Silverplatter-Variante einer Variante, die nur auf dem UltraNet-CD-Server lauffähig war, vorzuziehen. Allerdings ist zu betonen, daß oftmals keine Wahlmöglichkeit besteht und viele Hersteller von CD-Datenbanken bei der Retrievalprogrammierung technisch eigene Wege gehen, sich der Benutzer also auf kein einheitliches Abfragesystem einstellen kann und die Bibliothek bei der Aufarbeitung solcher Datenbanken im CD-Netzen oftmals diverse technische Klimmzüge unternehmen muß, um solch inhomogene Retrieval im Netzbetrieb lauffähig zu machen.

Zu Testzwecken wurde 1997 zusätzlich ein Knight-RidderServer installiert, da einige von den Fachbereichen der GHK gewünschte Datenbanken nur auf diesem System erhältlich sind. Mittlerweile wurde dieser auf NT basierende CD-Server der Firma Dialog in Kassel ausgebaut und beherbergt im Rahmen der landesweiten Konsortialverträge alle an den hessischen Hochschulen erworbenen Dialog-Datenbanken.
Die Abt. I'M hat die Administration dieses hessenweiten Servers übernommen.

Über die auf den CD- und WWW-Servern recherchierbaren Datenbestände (1994: 17 Datenbanken auf 21 CD's; 1997: 140 Datenbanken) informieren einige Web-Seiten der Bibliothek. Hier sind als Entscheidungskriterium für Weiterbeschaffungen neben Titel und Status der Datenbank auch die jeweilige Zugriffsform (UltraNet/WWW) und die Nutzungsdauer hinterlegt.

\section{Beteiligung an überregionalen Pro- jekten}

\section{WebDOC}

Traditionell sind Bibliotheken der Ort, an dem Literatur gesammelt, archiviert, katalogisiert und verfügbar gemacht wird. Allerdings hemmen auf längere Sicht nicht nur die immer knapper werdenden finanziellen Ressourcen diese Aufgabe; auch Archivspeicher und bibliothekarische Arbeitskraft lassen sich nicht im gleichen Maße vervielfachen, wie die Produktion von Literatur dies erfordern würde. Allein in den Naturwissenschaften gehen Schätzungen davon aus, daß sich alle 10 Jahre die Anzahl der wissenschaftlichen Publikationen verdoppelt. Einen Weg stellt die Absprache von Sondersammelgebieten der Bibliotheken untereinander dar, allerdings kann es kein sinnvoller Weg sein, notwendige Literatur stets über Fernleihe zu ordern, da diese Literatur die meiste Zeit nicht vor Ort benutzbar wäre und die Fernleihe mit ihren Dienstleistungen letztlich auch kostet.

Derzeit scheint die einzig mögliche Variante die elektronische Dokumentlieferung zu sein. Aufgrund des rapiden Preisverfalls bei Speichermedien ist die vollelektronische Archivierung kostengünstiger und platzsparender als die $\mathrm{Pa}$ piervariante. Dies gilt umso mehr bei Publikationen mit einem zeitlich begrenzten Informationswert. Auch der Versand der elektronischen Dokumente über weltweite Netze (Internet) ist schnell und preiswert.

Im Projekt WebDOC haben Endnutzer Zugang zu elektronischen Publikationen erhalten. Dazu wurde von PICA ein zentraler Server in Leiden errichtet, auf dem gemeinsam mit Partnerbibliotheken und Verlagen ein Online-Katalog (WebCat) zugänglich war. Die auf dem zentralen Server katalogisierten elektronischen Dokumente wurden als Volltexte auf Servern der Partnerbibliotheken gespeichert und archiviert. Die WebDOC-Datenbank war für Benutzer zugänglich, die in einer der beteiligten Teilnehmer-Bibliotheken angemeldet waren. Die GHB hatte als hessische Pilotbibliothek diesen Zugang zu WebDOC auf dem SparcCenter 20 installiert. Nach Abschluß (Nov. 1997) dieses Projektes kann resumiert werden, daß zwar wichtige Erfahrungen bei der Katalogisierung und Archivierung elektronischer Dokumente und der elektronischen Geldzahlung erworben wurden, jedoch die Nutzerschaft diesen Dienst bislang nicht im gewünschten Umfang angenommen hat. An dem Nachfolgeprojekt PiCarta von PICA wird sich die GHB ebenfalls beteiligen.

\section{Hessen-Media}

Online-Bibliothek. Im Rahmen der Initiative "Hessen Media" der Hessischen Landesregierung übernimmt die GHB die Durchführung und Koordination mehrer Projekte. Die Landesregierung fördert in diesem Zusammenhang Multimedia-Anwendungen in allen Bereichen der Gesellschaft. Über die Geschäftsstelle Hessenmedia (Wirtschaftsförderung Hessen, Investitionsbank AG - HLT) werden die zahlreichen Projekte gebündelt und dargestellt. Über 50 Projekte sind derzeit als förderungswürdig eingestuft. In dem ersten GHBProjekt werden die aus den o.a. Projekten hervorgehenden Publikationen, Print- oder Digitalpublikation, lektorats- und 


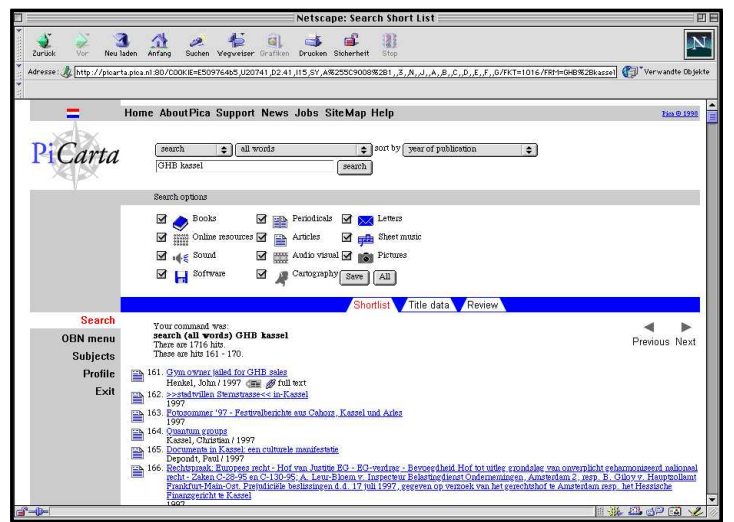

Abb. 19: PiCarta-Oberfläche

herstellungsmäßig betreut. Diese Publikationen sollen in einer aufzubauenden Online-Bibliothek digital aufbereitet und der Öffentlichkeit präsentiert werden. Erste Printpublikationen für die HLT liegen vor.

Benutzerfreundliche Oberfläche. Die Abt l'M projektiert im Rahmen der Landeslizenzen und des Projektes "Einheitliche Oberfläche" (Hessen-Media, s.u.) einen Dokumenten-Server für die hessischen Hochschulen aufbauen. Dieser soll, sobald die ersten Datenlieferungen (Back-Files der von der GHB verhandelten Landeslizenzen) Anfang 1999 eingetroffen sind, ans Netz gehen und mittels einer Retrieval-Software, für die eine Landeslizenz erworben werden konnte, für Mitglieder der Hessischen Hochschulen zugänglich sein. Die Software ist in der Lage (technisch wie rechtlich) 10 weitere dezentrale Hochschul-Server zusätzlich zu indizieren und somit unter einer Oberfläche zu erschließen.

\section{AG Landesweite Maßnahmen}

Im Rahmen des Sonderprogrammes "Wissenschaftliches Schrifttum" des Hessischen Ministeriums für Wissenschaft und Kunst wurden Gelder für landesweite Projekte 1999/ 2000 reserviert. Um die Projektmöglichkeiten auszuloten und entsprechende Vertragsverhandlungen zu führen, wurde eine Arbeitsgruppe berufen, die sich aus 5 Mitgliedern zusammensetzt. In mehreren Arbeitsgruppensitzungen wurden die eingeholten Angebote verschiedener Datenbank-Anbieter diskutiert und anschließend zu einer Gesamtliste zusammengestellt. Generell wurde folgendes festgehalten:

- Die Verträge sollen über alle Fachgebiete reichen, auch wenn dies anhand des unterschiedlichen Entwicklungsstandes bezüglich Online-Medien nicht immer ganz möglich ist.

- Um Bedürfnisse richtig abschätzen zu können und die Möglichkeit eines Weiterbezuges der Online-Medien durch die jeweilige Bibliothek zu fördern, sollen Eigenbeteiligungen eingeführt werden. Dies erhöht zudem die Anzahl der Angebote nicht unwesentlich.

- Es sollen die Verträge so gestaltet werden, daß über sog. „,back-files“ (möglichst PDF-Format) der Zugriff auch nach Ablauf der erworbenen Zugriffszeit auf eigenen Servern möglich ist.

Die im einzelnen beschlossenen Maßnahmen wurden arbeitsmäßig aufgeteilt. Über die Abt. I'M der GHB Kassel wurden folgende Konsortialverträge für die hessischen Hochund Fachhochschulen verhandelt und abgeschlossen:

- Academic Press (Online-Zeitschriften) [3]

- Dialog (verschiedene Datenbanken)[4]

- Karger (Online-Zeitschriften)[5]

- UMI (ABI-Inform Research)[6]

\section{Beschreiten neuer Wege: Gründung eines Verlages (KUP)}

Da die GHB über kein Sondersammelgebiet (SSG) verfügt, kann in längerfristiger Sicht eine finanzielle Sicherstellung der Bücherversorgung bei anhaltender Finanzknappheit nicht mit absoluter Sicherheit garantiert werden. Daher ist es derzeit umso wichtiger, Trends zu erkennen und in entsprechende Weichenstellungen umzusetzen. Deutlich dürfte in den letzten Jahren eine zunehmende Digitalisierung von text- und audiovisuellen Informationen geworden sein. Daher wird in naher Zukunft als weitere Aufgabe für Bibliotheken neben ihrer traditionellen Rolle als Sammler, Bewahrer und Ausleiher gedruckter Information die Vermittlung, ErschlieBung und Archivierung von digitalen Informationen immer mehr in den Vordergrund rücken.

Innerhalb des WEBDOC-Projektes wurde diese Online-Publishing-Idee bereits in Ansätzen umgesetzt. Parallel dazu existierten Ideen, angegliedert an die GHB einen Online-Publishing-Verlag (KUP - Kassel University Press) zu etablieren, der zum einen die Hochschulschriften aus Kassel verlegen, zum anderen jedoch auch für die anfallenden Dissertationen eine kostengünstige und im Rahmen der neuen Technologien effektiv zu handhabende Publikationsmöglichkeit darstellen sollte. Entgegen bereits existierender OnlinePublishing-Initiativen im wissenschaftlichen Bereich, wie dem Bibliotheks- oder Fachbereichs-Servern, dem DISS-Online-Projekt oder dem MEDOC/InterDOC-Projekt, war daran gedacht, eine Kooperation mit einem bestehenden Verlag zu initiieren. Bei dieser Kooperationslösung ergab sich neben der Online-Publishing-Variante auch die Möglichkeit, Dissertationen und Hochschulschriften bei Bedarf in gedruckter Form zu verlegen.

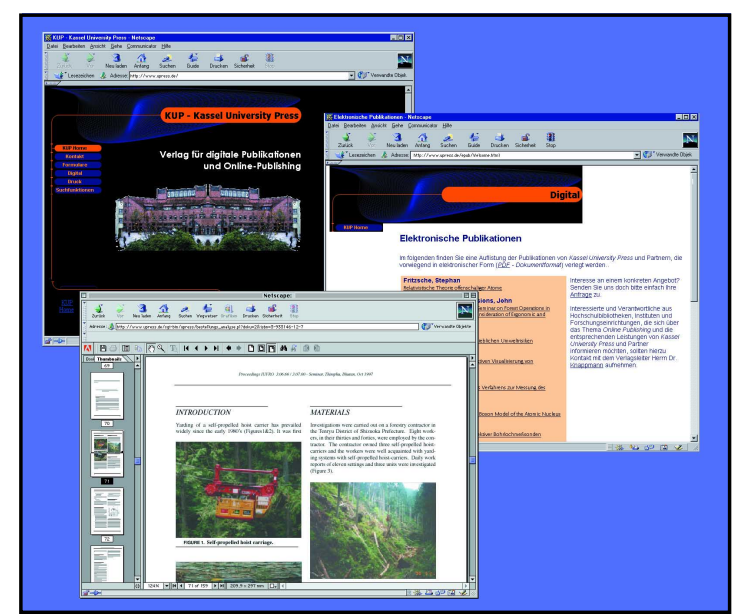

Abb. 20: WWW-Seiten von KUP

Im gegenwärtigen Stand des Projektes hat die GHB die entsprechende Hard- und Software-Installation (WWW-Server auf einer Alpha, NameServer für die eigene Domain) aufgebaut und administriert. Organisatorisch wird der Verlag, der durch einen eingesetzten Geschäftsführer repräsentiert wird, durch die beiden Gesellschafter resp. inre Vertreter vertreten. Innerhalb der vereinbarten zweijährigen Erprobungsphase fiel der Abt. I'M die technische Realisierung des Verlagsprojektes zu. Derzeit wird geprüft, unter welchen Bedingungen der Verlag weitergeführt werden kann.

Die Dienstleistungen der KUP beinhalten:

- umfassende verlegerische Betreuung bei der Aufbereitung elektronischer Publikationen (Volltextretrieval, Verschlagwortung, Implementation in einer PDF-

Datenbank, Aufbereitung von Abstract und Inhaltsverzeichnis in HTML, etc.)

- direkten Zugriff auf die in der KUP erschienenen digita- 
len Produkte (Möglichkeit, Kopie gegen Gebühr und/ oder als Printkopie)

Die Vorteile dieser Organisationsform liegen begründet in:

- schneller kostengünstiger Zugriffsmöglichkeit im WWW

- kostengünstiger Produktion

- lektorieller Betreuung

- Bedienung des Buchhandels und der Privatwirtschaft (u.a. ISBN- und CIP-Vergabe)

- Sicherung und Archivierung der digitalen Dokumente Entsprechende Informationen können unter "http.// www.upress.de" abgerufen werden.

\section{Zukunft}

Da derzeit eine weitgehende Dezentralisierung der ursprünglich einschichtigen Gesamthochschulbibliothek in zehn Bereichsbibliotheken mit einem relativ kleinen gemeinsamen Dienstleistungsbereich forciert wird, bleibt abzuwarten, wie sich diese Entwicklung auf den bibliothekarischen und DV-technischen Alltag für Mitarbeiten und Benutzer auswirkt.

\section{Anmerkungen}

[1] Zur Ausstattung der Hochschulen in der Bundesrepublik Deutschland mit Datenverarbeitungskapazität für die Jahre 1992 bis 1995, Empfehlungen der Kommission für Rechenanlagen der Deutschen Forschungsgemeinschaft, 1991, S. 36; Migration der deutschen Bibliotheksverbünde, Bibliotheksausschuß DFG, 1994; Deutsche Forschungsgemeinschaft: Elektronische Informations-Systeme und -Dienstleistungen in wissenschaftlichen Bibliotheken, September 1995, S. 1; Bericht und Empfehlungen der Enquete-Kommission "Entwicklung, Chancen und Auswirkungen neuer Informationsund Kommunikationstechnologien in Baden Württemberg" (Multimedia-Enquete), Drucksache 11/6400, Landtag von Baden Württemberg, 20.10.1995.

[2] Vgl. Jahresbericht 1997 der Gesamthochschul-Bibliothek Kassel, 1998.

[3] Eine Liste der Zeitschriften ist unter http://www.apnet.com/www/ap/maildate. $h$ tm einsehbar.

[4] Die Dialog-Datenbanken werden in Hessen über einen WWW-Server in Kassel administriert und distribuiert.

[5] Eine Liste der Zeitschriften ist unter http://www.online.karger.de/ einsehbar.

[6] Zugang über http://global.umi.com/pqdweb. 